\title{
Follow-up of the 9C survey: Initial results
}

\author{
Rosemary C. Bolton ${ }^{a}$ Garret Cotter $^{a}$ T.J. Pearson ${ }^{b}$ \\ Guy G. Pooley ${ }^{\mathrm{a}}$ A.C.S. Readhead ${ }^{\mathrm{b}}$ Julia M. Riley ${ }^{\mathrm{a}}$ \\ Elizabeth M. Waldram ${ }^{\text {a }}$ \\ a Astrophysics, Cavendish Laboratory, Cambridge CB3 OHE, UK \\ ${ }^{\mathrm{b}}$ California Institute of Technology, Pasadena, CA 91125, USA
}

\begin{abstract}
We present initial results from a follow-up of the $9 \mathrm{C}$ survey, complete to $25 \mathrm{mJy}$ at $15 \mathrm{GHz}$, designed to assemble and investigate a sample of young radiosources. We have made radio continuum maps of 111 sources at frequencies spanning $1.4-43$ $\mathrm{GHz}$, and classified them according to their radio size and spectral index between 1.4 and $4.8 \mathrm{GHz}$. We find that selection at $15 \mathrm{GHz}$ is twice as efficient at picking Gigahertz Peaked Spectrum (GPS) sources as selection at $\sim 5$ GHz. Optical followup has now begun; imaging of GPS and compact steep spectrum sources suggests that a significant fraction of the host galaxies have close companions or disturbed morphologies.
\end{abstract}

Key words: galaxies: active — galaxies: evolution — radio continuum : galaxies

\section{Introduction}

It is now widely believed, as evidenced by many contributions in these proceedings, that the onset of AGN activity in galaxies is closely linked to vigorous star-formation, possibly in the presence of mergers. However, the precise mechanism that causes radio sources to trigger remains the subject of lively debate - see, e.g.,(5) versus (8). To advance our understanding of this issue it is clearly necessary to assemble samples of radiosources at the earliest possible times in their evolution.

The Gigahertz Peaked Spectrum (GPS) and Compact Steep Spectrum (CSS) sources are now thought to be the progenitors of the larger, older members of the population. These young sources, often morphologically classified as Compact Symmetric Objects (CSOs), have indeed in a few cases been observed 
growing at sub-relativistic speeds by VLBI (6). Unfortunately it has been difficult to obtain flux-limited samples of GPS and CSS sources because largearea surveys at frequencies higher than $5 \mathrm{GHz}$ have been unavailable. Now, with the advent of cm-wavelength Cosmic Microwave Background (CMB) experiments, such surveys are being carried out to implement foreground source subtraction. The Cambridge Ryle Telscope (RT) is undertaking a survey at $15 \mathrm{GHz}$, the 9C survey (7), of CMB fields observed by the Very Small Array (VSA). Using 9C we have selected 156 sources on the basis of their $15 \mathrm{GHz}$ flux alone; these have all been followed up at frequencies from $1.4 \mathrm{GHz}$ to 43 $\mathrm{GHz}$. Radio frequency data reduction is now complete for two of the three sample fields and we present results for these 111 sources. This sub-sample is complete to $25 \mathrm{mJy}$ and covers a total area of 140 square degrees.

\section{Radio and optical observations and analysis}

The first three regions of $9 \mathrm{C}$ coincide with the VSA compact array fields, at $00^{h} 20^{m}+30^{\circ}, 09^{h} 40^{m}+32^{\circ}$ and $15^{h} 40^{m}+43^{\circ}$. Our sample is complete to $25 \mathrm{mJy}$ in the $00^{h}$, the $15^{h}$ field and for one-quarter of the $09^{h}$ field. The remaining three-quarters of the $09^{h}$ field is complete to $60 \mathrm{mJy}$. Continuum snapshot maps were made at 1.4, 4.8, 22 and $43 \mathrm{GHz}$ with the VLA and at 15 $\mathrm{GHz}$ with the RT between 2001 January and 2002 January. All observations for each region were made simultaneously to avoid problems introduced by source variability.

We have used the radio fluxes to classify each source according to the spectral index, $\alpha$ between 1.4 and $4.8 \mathrm{GHz}$ (we take $S \propto \nu^{-\alpha}$ ). We define three spectral classes: steep spectrum sources with $\alpha_{4.8}^{1.4} \geq 0.5$, flat spectrum sources with $-0.1 \leq \alpha_{4.8}^{1.4}<0.5$, and a third class containing those sources with $\alpha_{4.8}^{1.4}<-0.1$. The last of these includes the GPS sources displaying a peak in flux at 4.8, 15 or $22 \mathrm{GHz}$, and those still rising at $43 \mathrm{GHz}$, which are assumed to be extreme versions of the GPS sources. We also classify objects as compact if they have a radio size $\leq 2^{\prime \prime}$, and as extended otherwise. Of the 111 sources here, $55 \%$ are compact and $45 \%$ are extended in the radio. The numbers of sources in each class are summarised in Table 1.

It is already well established for samples selected at lower radio frequencies that extended radio sources have steeper spectra than compact sources (see, e.g. Peacock and Wall (4), PW hereafter), and the trend continues for this sample. Plotting the distribution of spectral index for the two size classes (Figure 1) clearly shows this tendency: the median values of $\alpha_{4.8}^{1.4}$ are 0.21 and 0.76 for the compact and extended radio sources respectively. To examine how useful our selection process is as a means of finding GPS sources we compare our sample to PW, who studied a sample of bright $\left(S_{2.7} \geq 1.5 \mathrm{Jy}\right)$ radio 
Table 1

\begin{tabular}{|l|l|l|l|}
\hline & Compact sources & Extended sources & Total \\
\hline Steep spectrum & 19 & 37 & 56 \\
Flat spectrum & 23 & 11 & 34 \\
Peaked spectrum & 19 & 2 & 21 \\
\hline Total & 61 & 50 & 111 \\
\hline
\end{tabular}

Numbers of sources of different classes for the $9 \mathrm{C}$ subsample presented here.
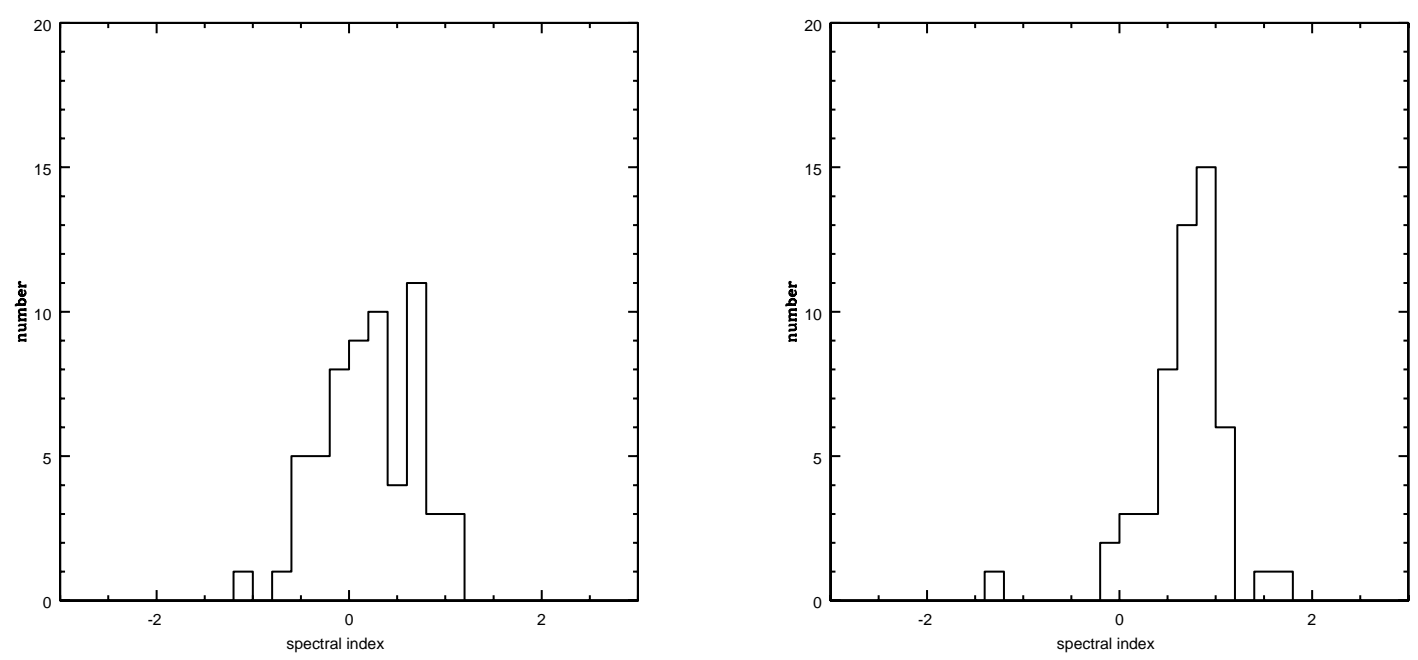

Fig. 1. Binned histograms of spectral index between 1.4 and $4.8 \mathrm{GHz}$ for the compact (left) and the extended (right) radio sources (right) (bin size 0.2).

sources selected at $2.7 \mathrm{GHz}$. By combining our flat and peaked sources into the same class (i.e $\alpha<0.5$ ) we can directly compare our sample with PW. The percentages of sources falling into each class in the two samples are shown in table 2. Our sample contains a lower fraction of steep-spectrum sources than PW, but roughly the same fraction of the compact sources (note, though, that PW calculate $\alpha$ between 2.7 and $5 \mathrm{GHz}$, and so will be more likely to classify sources with standard convex spectra as steep-spectrum). PW did not make any distinction between flat-spectrum and GPS sources, but O'Dea (3) summarised the frequency of occurence of GPS and compact steep spectrum (CSS) sources for flux-limited samples taken at around $5 \mathrm{GHz}$. Using the values given by O'Dea and (1), along with the values from PW, the numbers of extended steep spectrum, CSS, flat spectrum and GPS sources a sample selected at $\sim 5 \mathrm{GHz}$ should contain can be estimated. These approximate percentage values, and those of this work, are given in table 3 . We find roughly twice as many GPS sources as in samples selected at $\sim 5 \mathrm{GHz}$, but only about half as many CSS sources.

A programme of optical imaging and spectroscopy is underway to obtain iden- 


\begin{tabular}{|l|l|l|l|l|}
\hline & Sample & Compact sources & Extended sources & Total \\
\hline Steep spectrum & This work & 17 & 33 & 50 \\
$(\alpha \geq 0.5)$ & PW & 22 & 48 & 70 \\
\hline "Flat" spectrum & This work & 38 & 12 & 50 \\
$(\alpha<0.5)$ & PW & 27 & 3 & 30 \\
\hline Total & This work & 55 & 45 & \\
Total & PW & 49 & 51 & \\
\hline
\end{tabular}

Table 2

Percentages of sources in different classes for this work and the PW sample.

\begin{tabular}{|l|l|l|}
\hline Selection Frequency & $\sim 5 \mathrm{GHz}$ & $15 \mathrm{GHz}$ \\
\hline Extended Steep Spectrum & 40 & 33 \\
\hline Compact Steep Spectrum & 30 & 17 \\
\hline Flat Spectrum & 20 & 31 \\
\hline Giga-Hertz Peaked Spectrum & 10 & 19 \\
\hline
\end{tabular}

Table 3

Approximate percentages of different source classes for a typical $\sim 5 \mathrm{GHz}$ sample and this work.

tifications and redshifts for the compact sources. To date (2003 January) we have obtained imaging with the Palomar $60^{\prime \prime}$ telescope of roughly two-thirds of the sample, including all targets fainter than $R_{\mathrm{AB}}=19.2$. While we caution that this work is still incomplete, we note that the initial results suggest that many of the compact sources have close companions or disturbed morphology at optical wavelengths. We find that almost all the low- $z$ host galaxies imaged to date have faint companions within $\sim 10$ arcsec. As we move to more distant host galaxies, where our imaging programme is thus far insufficiently deep to detect very faint companions, several noticeably turbulent environments have been discovered (Fig. 2).

It is clearly now essential, in the light of the debate over major versus minor interactions as the trigger for radiosource activity, that we quantify these optical observations for the complete sample and compare with control samples of both larger radiosources and quiescent ellipticals.

\section{$3 \quad$ Discussion and summary}

In the standard picture of GPS sources, the energy density of the synchrotron plasma in the lobes is sufficiently high that they remain optically thick to 


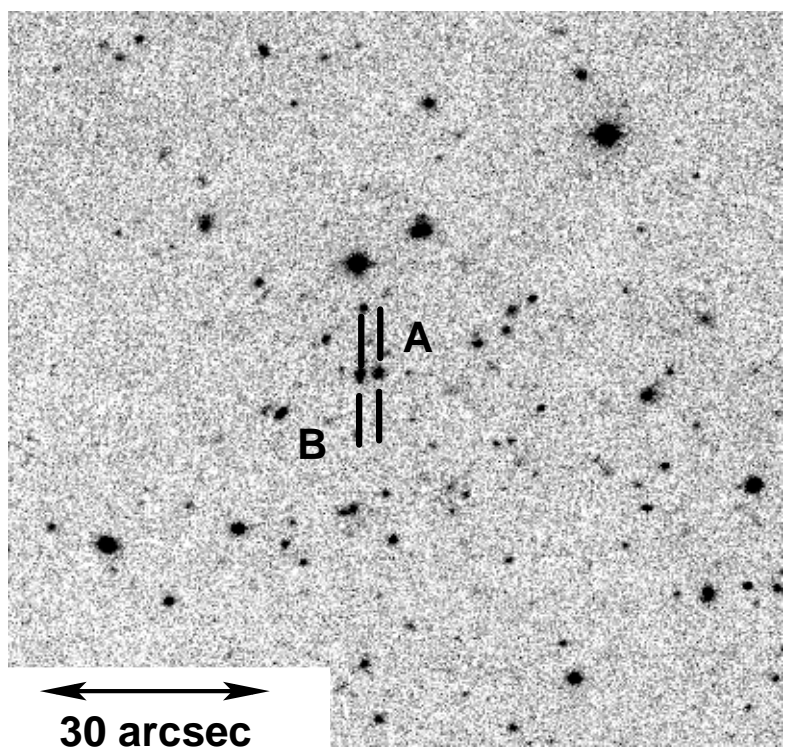

Fig. 2. 3600-s Palomar 60" I-band image of a $z=0.9$ system containing a $9 \mathrm{C}$ CSS galaxy. Both the radiogalaxy, A, and its near companion, $\mathrm{B}$, have disturbed morphologies and show bright AGN emission lines. There is a clear excess of faint galaxies centred on the radiosource; we speculate that this is a dynamically unrelaxed, possibly merging, cluster.

higher radio frequencies (2), with the spectral peak indicating the frequency at which the lobe plasma becomes optically thin. As the source grows to kpc size, the peak in the spectrum remains, but falls to $\mathrm{MHz}$ frequencies and below, and a CSS source is observed.

Almost all previously known GPS sources have peak frequencies of order $1-5$ GHz. In our sample, however, we have found a significant number of sources which peak near the selection frequency. Of the 21 peaked spectrum sources, 13 peak at $15 \mathrm{GHz}$ or above (three are still rising at $43 \mathrm{GHz}$ ). Two examples of these GPS spectra are shown in Figure 3. If the known trend continuesthat the peak frequency increases as sources are seen younger and youngerwe speculate that these very high frequency peakers are likely to be very young sources indeed. Efforts are underway to develop models which track the dynamical evolution and synchrotron spectrum to very early times.

We have found that selecting at $15 \mathrm{GHz}$ is roughly twice as efficient at picking out GPS sources than at $\sim 5 \mathrm{GHz}$, which makes it a valuable method of creating flux-limited samples of such objects. The 9C survey, and other forthcoming cm-wave surveys, are thus useful not only for studying the radio source population at high radio frequencies as a whole, but also as a means of singling out what are potentially the very youngest sources for further study. Our optical imaging is now uncovering evidence suggesting that a significant fraction of the host galaxies may be interacting. In the near future we hope to quantify these processes with control samples of both larger radiosources and 

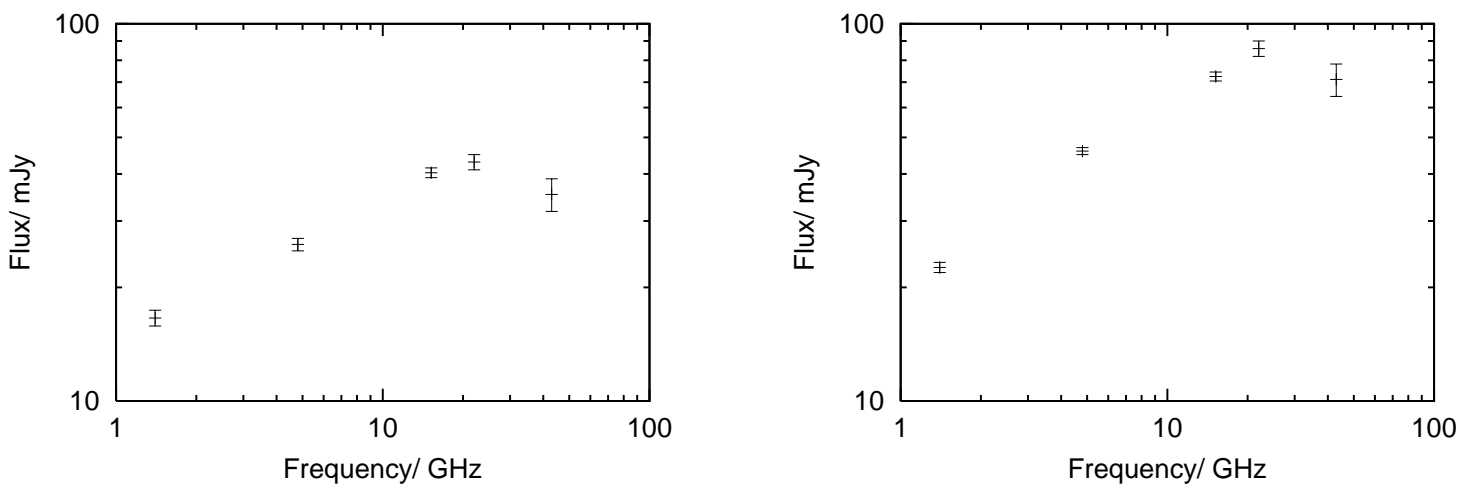

Fig. 3. Radio spectra for two GPS sources peaking near $22 \mathrm{GHz}$.

non-AGN ellipticals. Plans are also in place to select a sample at a fainter flux limit, so that we may obtain access to sources matched in luminosity over a significant redshift range. We are hopeful that detailed studies of this sample and similar ones selected at high frequency will allow us both to model precisely the earliest stages of radiosource evolution, and to determine the exact causal connection between galaxy-galaxy interactions and the triggering of the radiosource.

\section{Acknowledgements}

RCB thanks PPARC for a research studentship. The Ryle Telescope is funded by PPARC. The VLA is operated by the National Radio Astronomy Observatory, which is a facility of the National Science Foundation, operated under cooperative agreement by Associated Universities, Inc.

\section{References}

[1] V. K. Kapahi (1981) A\&AS. 43 381-394.

[2] K. I. Kellermann (1966) Australian J. Phys. 19195

[3] C. P. O'Dea (1998) PASP. 110493.

[4] J. A. Peacock \& J. V. Wall (1981) Mon.Not.R.astr.Soc. 194 331-349.

[5] E. S. Perlman, J. T. Stocke, J. E. Conway, \& C. S. Reynolds. (2000) American Astronomical Society Meeting. 197

[6] G. B. Taylor, J. M. Marr, T. J. Pearson, \& A. C. S. Readhead. (2000) The Astrophysical Journal. 5411

[7] E. M. Waldram, G. G. Pooley, K. J. B. Grainge, M. E. Jones, R. D. E. Saunders, P. F. Scott \& A. C. Taylor Mon.Not.R.astr.Soc. (submitted)

[8] A. S. Wilson, \& E. J. M. Colbert.(1995) Bulletin of the American Astronomical Society. 27830 